\title{
S? \\ Photonic band-gap formation, light diffusion, and localization in photonic amorphous diamond structures
}

\author{
Shigeki Imagawa, ${ }^{1}$ Keiichi Edagawa, ${ }^{1, *}$ Keisuke Morita, ${ }^{1}$ Toshiki Niino, ${ }^{1}$ Yutaka Kagawa, ${ }^{2}$ and Masaya Notomi ${ }^{3,4}$ \\ ${ }^{1}$ Institute of Industrial Science, The University of Tokyo, Komaba, Meguro-ku, Tokyo 153-8505, Japan \\ ${ }^{2}$ Research Center for Advanced Science and Technology, The University of Tokyo, Komaba, Meguro-ku, Tokyo 153-8505, Japan \\ ${ }^{3}$ NTT Basic Research Laboratories, NTT Corporation, 3-1, Morinosato-Wakamiya Atsugi, Kanagawa 243-0198, Japan \\ ${ }^{4}$ CREST, Japan Science and Technology Agency, Honmachi, Kawaguchi, 332-0012, Japan
}

(Received 30 June 2010; published 17 September 2010)

\begin{abstract}
We report experimental and numerical studies on photonic band-gap (PBG) formation and light propagation in a recently proposed unique photonic structure, "photonic amorphous diamond (PAD)." PADs have been fabricated in a microwave regime, and the formation of a full three-dimensional (3D) PBG has been substantiated experimentally. This proves unambiguously that periodicity is not essential to the realization of a 3D-PBG, contrary to the common belief. The 3D-PBG has been demonstrated to be completely isotropic, regardless of the light polarization direction, which, in principle, cannot be realized in conventional photonic crystals. In passbands, the PAD has exhibited diffusive light propagation, where the scattering strength increases significantly as the frequency approaches the band edge, indicating a precursor of light localization. Localized states have indeed been identified at the band edges by a numerical calculation. Numerical studies have also indicated that the picture of dielectric and air bands in conventional photonic crystals can be applied to the PBG formation in PAD as well. These findings provide different insights into the physical origin of PBGs and issues such as light diffusion and localization in photonic materials.
\end{abstract}

DOI: 10.1103/PhysRevB.82.115116

\section{INTRODUCTION}

In 1987, Yablonovitch ${ }^{1}$ and John ${ }^{2}$ proposed the idea that a three-dimensional (3D) photonic band gap (PBG), in which electromagnetic wave propagation is forbidden in all directions, can be realized in artificial 3D periodic dielectric structures. Since then, these "photonic crystals" have attracted considerable attention and have been studied extensively because of their great potential for applications to strong light confinement and manipulation of light propagation, ${ }^{3}$ which might enable us to realize novel optic devices. Because Bragg scattering due to lattice periodicity has been considered to be the main origin of gap formation, it was commonly believed that lattice periodicity is indispensable for the realization of 3D-PBGs. However, in contrast, it was indicated recently by numerical calculations that a 3D amorphous structure can form a sizable $3 \mathrm{D}-\mathrm{PBG},{ }^{4}$ despite the complete absence of lattice periodicity.

The proposed amorphous structure consists of a random network of dielectric rods with a diamond-like local tetrahedral configuration and is therefore named "photonic amorphous diamond (PAD)" [see Fig. 1(a)]. More specifically, the PAD structure is based on the concept of a "continuous random network (CRN)" that was originally developed to describe the atomic arrangement of amorphous $\mathrm{Si}$ or Ge. ${ }^{5}$ For comparison, a photonic crystalline diamond (PCD) structure, which is constructed by connecting tetrahedral bonds in the crystalline diamond structure with dielectric rods, is shown in Fig. 1(b). This PCD structure is known to be the best 3D-PBG structure; it exhibits the largest 3D-PBG among all the photonic crystals studied thus far. ${ }^{6,7}$ PAD and PCD can be regarded as the photonic versions of amorphous and crystalline $\mathrm{Si}$, respectively, in electronic systems.

In general, CRN structures are constructed using the socalled Wooten-Winer-Weaire algorithm. ${ }^{5,8}$ In this algorithm,
PACS number(s): 42.70.Qs

we start from a crystalline diamond structure with the tetrahedral bonding. It is then randomized by a large number of bond transpositions. Here, each bond transposition is done so that it does not generate a coordination number other than four. After each transposition, all atoms are relaxed with an assumed interatomic potential favoring the ideal local tetrahedral configuration. For the CRN structure used for the construction of our PAD, an improved algorithm is adopted, where we start from a random configuration, instead of a crystalline diamond structure. ${ }^{8}$ This guarantees that the constructed structure is not contaminated by any memory of the initial crystalline structure. Therefore, although both the PAD and PCD structures comprise fourfold connections, the global network topology in the PAD structure is completely different from that in the PCD structure. Thus, the PAD struc-
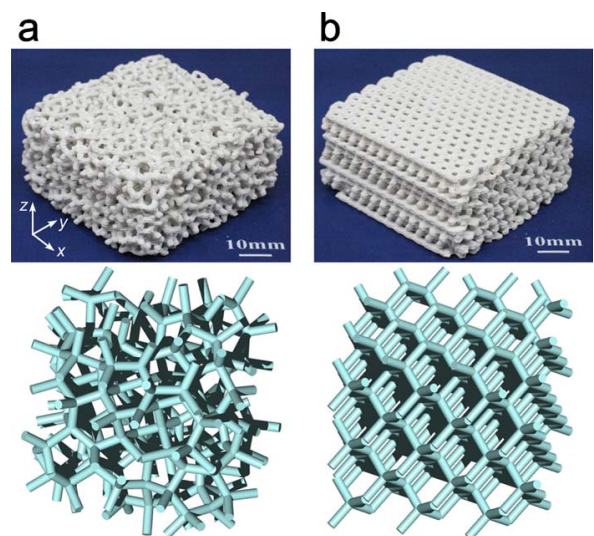

FIG. 1. (Color online) Experimental photonic structures and their computer graphics (CG) images of (a) PAD and (b) PCD. The rod length and radius are $d \approx 3 \mathrm{~mm}$ and $r \approx 0.78 \mathrm{~mm}(=0.26 d)$, respectively, for the fabricated structures. For clarity, the rod radius is $r=0.15 d$ for the CG images. 
ture is essentially different from the disordered PCD structures that preserve the network topology of a PCD. In general, such disordered photonic crystals preserve the original periodicity as a residue in the average structure because of the preserved network topology of the PCD, and the surviving band gap may be attributable to the residual periodicity. In contrast, the PAD has no such residual periodicity and its band-gap formation should not be due to periodicity.

Light propagation in random media has been a longstanding subject in optics. ${ }^{9,10}$ In random media, light undergoes multiple scattering. Then, light propagates diffusively as long as the scattering is not very strong. As the scattering intensifies, i.e., the scattering mean-free path $l$ reduces, light interference intensifies, leading to a halt of diffusive light propagation. This phenomenon is known as the Anderson localization of light. ${ }^{9,11-19}$ The criterion for the emergence of Anderson localization is given as $2 \pi l / \lambda \approx 1$ (Ioffe-Regel condition $^{20}$ ), where $\lambda$ denotes the light wavelength. Thus far, several systems ${ }^{15-18,21,22}$ have been reported to be close to satisfying this criterion and some of them have been claimed to exhibit light localization. However, the signatures of localization can be similar to those of absorption, and this fact generally makes it difficult to unequivocally prove the realization of light localization. ${ }^{16,19}$

In this paper, we report detailed experimental and numerical studies on PBG formation and light propagation in PAD. The purpose of the studies is to substantiate experimentally the 3D-PBG formation in PAD and to reveal characteristic features of photonic eigenstates and light propagation properties of PAD. This paper is organized as follows. In Sec. II, the experimental and calculation methods are explained. In Sec. III, the results are described. Here, the results related to light propagation properties are first presented in Sec. III A, and then, those of numerical calculations of photonic eigenstates are presented in Sec. III B. These results are discussed in Sec. IV. Finally, Sec. V is devoted to concluding remarks.

\section{METHODS}

PAD and PCD structures were fabricated by a selective laser sintering method. ${ }^{23}$ The PCD structures were fabricated for comparative experiments. Figures 1(a) and 1(b) show examples of the fabricated PAD and PCD structures, respectively. Mixtures of nylon (PA12) powders (typically $50 \mu \mathrm{m}$ in diameter) and $\mathrm{TiO}_{2}$ powders $(75-100 \mu \mathrm{m}$ in diameter) were used for fabrication. $\mathrm{TiO}_{2}$ powders were added to increase the refractive index $n$ of the rods. The rod had a porosity of approximately $40 \%$. The fabricated structures were soaked in water for $24 \mathrm{~h}$, dried for $2 \mathrm{~h}$, and cooled to $268 \mathrm{~K}$. This resulted in the formation of ice in the pores of the rods, leading to a further increase in the refractive index $n$. Ice is suitable for increasing $n$ without increasing the extinction coefficient $\kappa$. The final $n$ value of the rods was determined experimentally to be 3.0. The volume fraction of the rod in the constructed structures is $22 \%$ and the rest is air. Then, the volume-weighted average $\bar{n}$ of refractive index is approximately 1.4. The values of $\kappa$ of nylon, $\mathrm{TiO}_{2}$, and ice are 3 $\times 10^{-2}, 2 \times 10^{-3}$, and $3 \times 10^{-3}$, respectively, in the gigahertz range. This indicates that the $\kappa$ value of the rods is approxi-

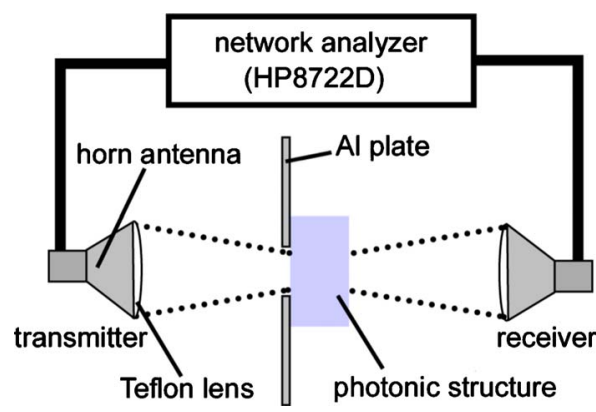

FIG. 2. (Color online) A schematic illustration of the experimental setting for microwave transmission measurements.

mately $2 \times 10^{-2}$. Then, the $(1 / e)$-absorption length $L_{a}$ in the structure can be estimated to be $1.7 \mathrm{~m}$ for $30 \mathrm{GHz}$ microwaves.

Each rod is $d \approx 3 \mathrm{~mm}$ in length and $r$ $\approx 0.78 \mathrm{~mm}(0.26 d)$ in radius. The size of the fabricated structures is approximately $x \times y \times z=70 \times 70 \times 35 \mathrm{~mm}^{3}$ $\left(23.3 \times 23.3 \times 11.7 d^{3}\right)$. Microwave signals were incident on the $x-y$ plane, along the $z$ axis. In the design of PAD, we used the CRN structure constructed and provided by Barkema and Mousseau. ${ }^{8}$ This CRN structure consists of a periodic arrangement of a cubic supercell with the size $(11.5 d)^{3}$. Three PAD samples with different orientations were fabricated. These were cut out of the infinite periodic supercell structure. The $z$ directions of the three samples are along [100], [110], and [111] of the cubic supercell, respectively. For PCD, two samples with different orientations were fabricated: samples with the $z$ direction along [100] and [111] of the diamond crystal.

For microwave transmission measurements, we employed a free-space method using a vector network analyzer (HP Model 8722D, Agilent Technologies) in the frequency range 18-35 GHz in the configuration schematically shown in Fig. 2. Conical horns with Teflon microwave lenses were positioned $163 \mathrm{~mm}$ in front of and behind the sample. Linearly polarized microwave radiation was emitted from one horn (transmitter), and the parallel- and cross-polarized components of the transmitted field were detected using the other horn (receiver) by changing the relative orientations of the mode selectors attached to the two horns. The transmittances $T_{p}$ and $T_{c}$ of the parallel- and cross-polarized components, respectively, were obtained by normalizing the measured intensities to the intensity of the parallel component measured before mounting the sample. The background level was evaluated to be $3 \times 10^{-6}$. 10-20 measurements were performed by changing the $x-y$ positions of the sample to obtain the averaged spectra. Not only the intensity but also the phase of the transmitted waves, i.e., the complete complex transmission spectra were measured. They were used for the calculation of the response to an input pulse. Frequency dependence of group delay was also evaluated from the phase data.

The spectral intensities, which represent the photonic density of states (PDOS), and the eigenmodes for PAD and PCD were calculated by a finite-difference time domain (FDTD) spectral method originally developed by Chan et al. ${ }^{24}$ In this method, we start from a random electromagnetic field and 
calculate its time evolution. The time evolutions at each position is then Fourier transformed into frequency domain to obtain PDOS and eigenmodes. As in the experimental fabrication, we used the CRN structure of Bakema and Mousseau $^{8}$ for the design of PAD, which consists of a periodically arranged cubic supercell of size $(11.5 d)^{3}$. For PCD, we used a crystalline diamond structure with a fictitious supercell $(5 a)^{3}=(11.5 d)^{3}$, where $a=4 d / \sqrt{3}$ denotes the lattice constant of the crystalline diamond. Using the fictitious supercell structure for PCD enables us to compare the results obtained under exactly the same conditions for PAD and PCD. We modeled the PAD and PCD structures by connecting the tetrahedral bonds with dielectric rods in a background of air. The rod radius was $r=0.26 \mathrm{~d}$. The refractive index of rods was $n=3.0$ and the absorption was neglected, i.e., $\kappa$ $=0$.

For the detailed calculation procedures, we followed Ref. 4, except for a few points described below. In this study, we adopted a Blackman function as the window function in Fourier transformation, instead of a rectangular function used in Ref. 4. Due to the smoothness of the Blackman function, we could obtain much higher precision in evaluating the amount of photonic states possibly existing in the gap. The number of sampling points was larger than that in ref. 4; we selected $42^{3}$ evenly distributed points with the interval of $0.243 d$ for PAD and PCD. For PCD, the photonic band structure was calculated also by a plane-wave expansion method using "BANDSOLVE" (RSoft Design Group, Inc.) to evaluate the frequency ranges of the band gaps in particular directions.

\section{RESULTS}

\section{A. Light propagation properties}

Figures 3(a) and 3(b) present the microwave transmittances $T_{p}$ and $T_{c}$ for PCD, respectively. Frequency is shown in the unit $c / d$ ( $c$ : speed of light). The blue and red spectra (lighter and darker lines, respectively) are for the microwave incident directions along [100] and [111], respectively. The spectra exhibit large decreases reaching $10^{-4}-10^{-5}$ in the transmittance. In Fig. 3(c), the calculated spectral intensity, which represents PDOS, is shown. In this calculated spectra, a large dip is clearly seen, which corresponds to a full $3 \mathrm{D}-\mathrm{PBG}{ }^{4}$ The gap width to the midgap frequency ratio $\Delta f / f_{c}$ is $22 \%$. The dip positions in the measured spectra for the [100] (blue) and [111] (red) incidences are slightly different from each other, and also each of them is slightly different from the calculated dip position in Fig. 3(c). This indicates an anisotropic gap formation. Figure 4 shows the photonic band structure calculated for the PCD by a planewave expansion method. The dip positions for the [100] and [111] incidences are expected to correspond to the band gaps in the $\Gamma-\mathrm{X}$ and $\Gamma$-L directions, respectively. The calculated band-gap positions in Fig. 4 are indicated by blue and red bars in Figs. 3(a) and 3(b), which indeed agree quite well with the observed dip positions. The overlapping frequency range of the dips for the two incidences roughly corresponds to the 3D-PBG.

Figures 5(a) and 5(b) show the transmittances $T_{p}$ and $T_{c}$ for PAD, respectively. The six curves are for the three

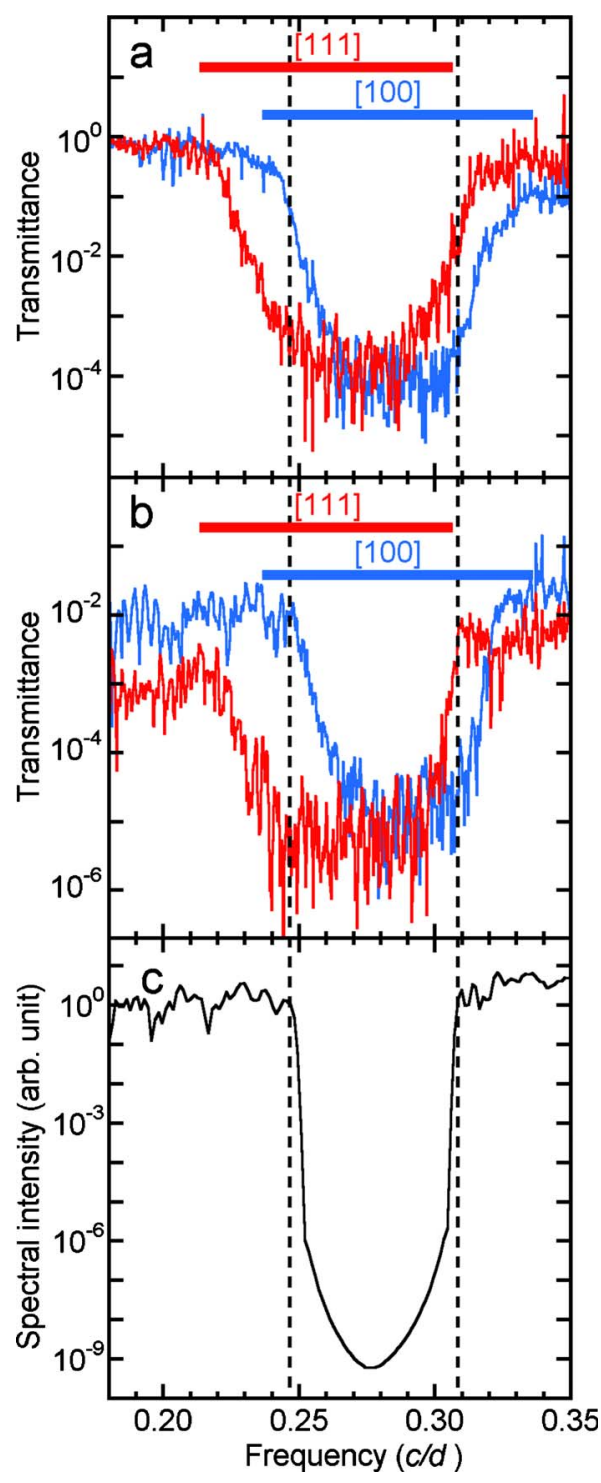

FIG. 3. (Color online) Microwave transmission spectra of (a) $T_{p}$ and (b) $T_{c}$ for PCD. The blue and red spectra (lighter and darker lines, respectively) are for the microwave incident directions along [100] and [111], respectively. The calculated spectral intensity, which represents PDOS, is shown in (c). The blue and red bars in (a) and (b) indicate the gap positions in the $\Gamma$-X and $\Gamma$-L directions, respectively, deduced by a photonic band-structure calculation using a plane-wave expansion method (Fig. 4).

samples with different orientations, and for two mutually orthogonal directions of the incident microwave polarizations for each sample. In Fig. 5(c), the calculated spectral intensity is shown, where a large decrease is clearly seen, corresponding to a full 3D-PBG. ${ }^{4}$ The gap width to the midgap frequency ratio $\Delta f / f_{c}$ is $13 \%$. Here, we first examine the spectra of $T_{c}$ in Fig. 5(b). All the six spectra exhibit clear dips and the frequency ranges of the dips coincide well. This is in contrast to the case of PCD in which the two spectra for the different incident directions show the dips in different frequency ranges. In addition, the frequency ranges of the dips in Fig. 5(b) agree well with that of the calculated 3D-PBG in Fig. 5(c). These facts indicate that the measured dips in $T_{c}$ in 


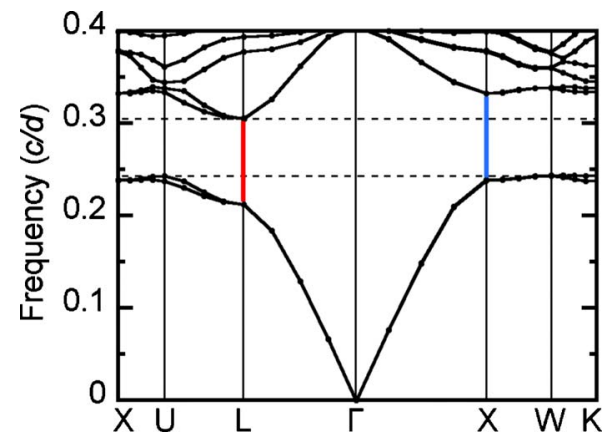

FIG. 4. (Color online) Photonic band structure of PCD calculated by a plane-wave expansion method. The blue and red bars indicate the band gaps in the $\Gamma-\mathrm{X}$ and $\Gamma$-L directions, respectively. The dashed lines indicate the full 3D-PBG.

Fig. 5(b) represent an isotropic 3D-PBG in PAD. However, we notice a peculiar feature in the spectra of $T_{p}$ for PAD in Fig. 5(a): the decrease in $T_{p}$ is not restricted to the frequency range of the calculated 3D-PBG in Fig. 5(c). To conclude the experimental substantiation of the 3D-PBG formation in our PAD, we have to clarify the reason for the peculiar feature in $T_{p}$. We have indeed succeeded in clarifying it, as follows.

In our experimental setting, the receiver has a relatively small receiving angle: approximately $-10 \mathrm{~dB}$ attenuation at $15^{\circ}$ deviation. This indicates that the parallel-polarized component $T_{p}$ reaching the receiver corresponds predominantly to the microwaves traveling directly from the transmitter to the receiver without being scattered; i.e., the ballistic component. On the other hand, the cross-polarized waves should have experienced scatterings that change the polarization direction; the component $T_{c}$ corresponds to the waves that were multiply scattered, ultimately returning to the direct path between the transmitter and receiver; i.e., the diffusive component. It should be noted that this does not represent the total intensity of the diffusively transported waves; rather it corresponds to a small fraction of them because the diffusively transported waves diverge in various directions. In principle, at the low-frequency limits with sufficiently large wavelengths, the wave propagation should be entirely ballistic. In the case of PAD, $T_{p}$ (i.e., the ballistic component) in Fig. 5(a) begins decreasing at $f \approx 0.20 c / d$, which is far from the band edge, rapidly declining to $10^{-3}$ at the band-edge frequency of $f \approx 0.26 \mathrm{c} / \mathrm{d}$. Above the gap, the transmission remains at $10^{-3}-10^{-2}$. This is in contrast to $\mathrm{PCD}$, in which the wave propagation is dominantly ballistic within the entire passbands, as evidenced by large $T_{p}$ values close to unity in Fig. 3(a).

The large reduction in the ballistic component of PAD should be due to the scattering that changes the propagation and polarization directions of the microwaves. Here, the scattering mean-free path $l$ can be estimated by the relation $T_{p}$ $=\exp (-L / l)(L$ : sample thickness $) .{ }^{10,18}$ The estimated $l$ is plotted in Fig. 6. At the lowest frequency of $f=0.18 c / d, l$ $\gg d$ holds and the microwave transport is dominantly ballistic. As the frequency increases, $l$ decreases to approach $d$ and the diffusive transport with multiple scattering progressively becomes dominant. At the band edge of $f \approx 0.26 \mathrm{c} / d, l$ reaches $1.7 d \approx 5 \mathrm{~mm}$, and the transport is diffusive with

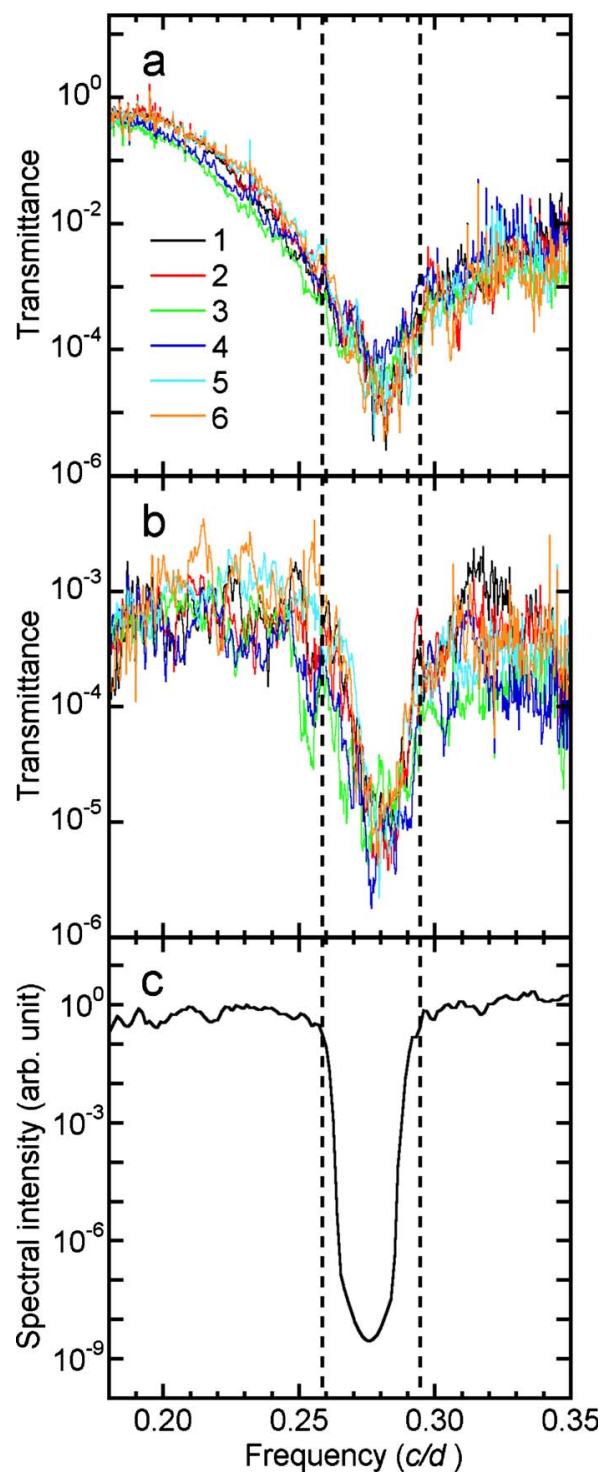

FIG. 5. (Color online) Microwave transmission spectra of (a) $T_{p}$ and (b) $T_{c}$ for PAD. The calculated spectral intensity, which represents PDOS, is shown in (c). The six curves in (a) and (b) are for three samples with different orientations, and for two mutually orthogonal directions of the incident microwave polarizations for each sample. The microwave incident directions of the three samples (A, B, and C) are along [100], [110], and [111] of the cubic supercell of $\mathrm{PAD}$, respectively. The microwave polarization directions are along (line 1) [010] and (2) [001] for A, (3) [001] and (4) [1 $\overline{1} 0]$ for B, and (5) $[\overline{1} \overline{1} 2]$ and (6) $[1 \overline{1} 0]$ for $\mathrm{C}$.

strong scattering. Above the gap, $l \approx 5-8 \mathrm{~mm}$, indicating that the diffusive transport is dominant. The quantity $2 \pi l / \lambda$ is estimated to be as low as 3 , where we use $\lambda=10 \mathrm{~mm}$ at 30 $\mathrm{GHz}$ in vacuum for a rough estimation. This is only slightly larger than the threshold value of unity in the Ioffe-Regel condition.

In Figs. 7(a) and 7(b), the spectral phases $\varphi$ of the parallel- and cross-polarized components of transmission are plotted for PAD and PCD, respectively. For PCD, the microwave incident direction is along [111]. Here, the phase values are given as the phase differences from the reference 


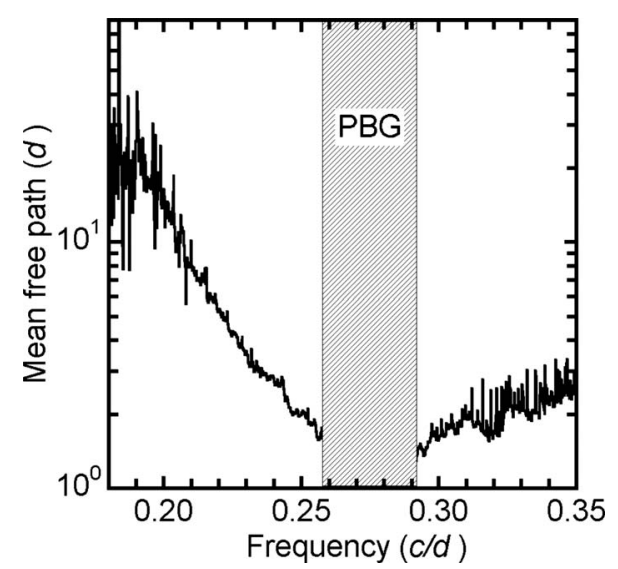

FIG. 6. Frequency dependence of the scattering mean-free path deduced from the decay of ballistic transmission.

measured before mounting the sample. We have added $2 \pi n$ ( $n$ : an arbitrary integer) to the measured phase within the range $[0,2 \pi]$ so that its frequency dependence becomes continuous. We failed to evaluate the phase in the gap frequency range due to a large scatter in the measured phase data; the relative vertical shift between the two curves below and above the gap in Figs. 7(a) and 7(b) has no physical meaning. In Figs. 7(c) and 7(d), we have evaluated the group delay given by $T_{\text {delay }}=-\frac{1}{2 \pi} \frac{d \varphi}{d f}$ for PAD and PCD, respectively. First, $T_{\text {delay }}$ of the parallel component for PAD in Fig. 7 (c) is small and almost constant. This should be a characteristic feature of the ballistic component in randomly scattering systems. In conventional photonic crystals, $T_{\text {delay }}$ generally increases as the frequency approaches the band edge
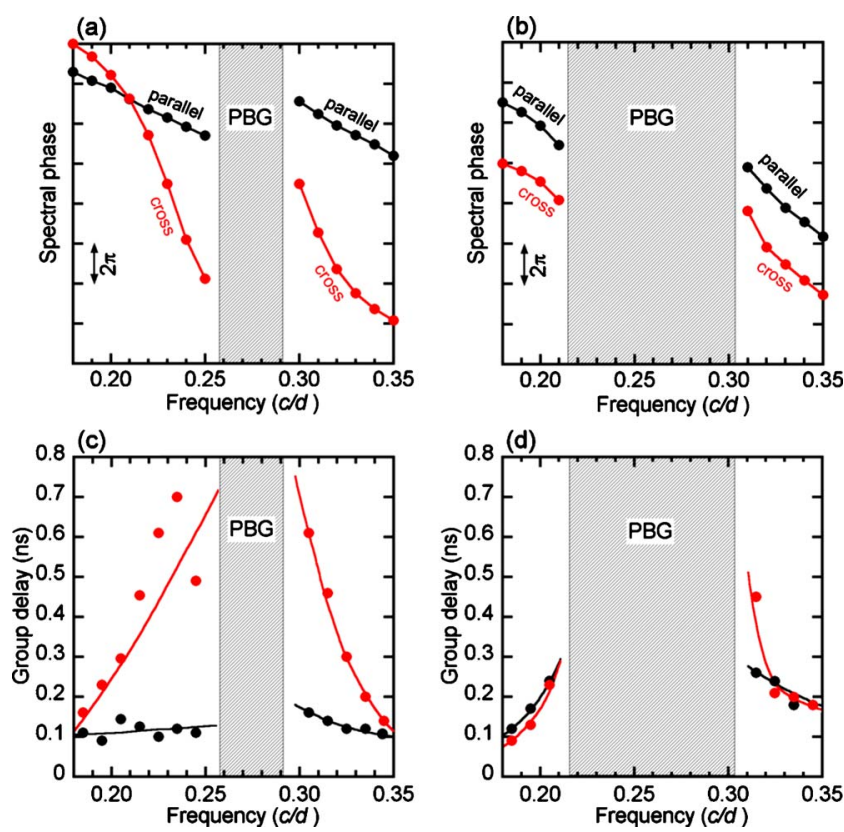

FIG. 7. (Color online) Spectral phases $\varphi$ of the parallel- and cross-polarized components for (a) PAD and (b) PCD, and group delays $T_{\text {delay }}$ calculated from the phases $\varphi$ using the relation $T_{\text {delay }}=-\frac{1}{2 \pi} \frac{d \varphi}{d f}$ for (c) PAD and (d) PCD. For PCD, the microwave incident direction is along [111].

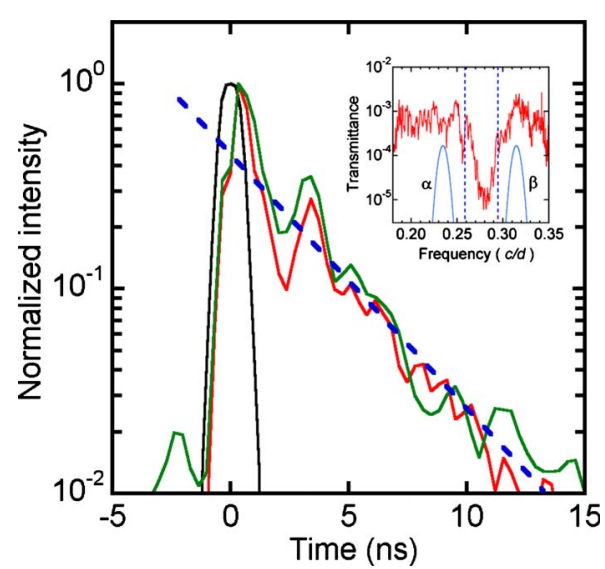

FIG. 8. (Color online) Intensity profiles (green and red) of transmitted pulses responding to input Gaussian pulses given by the spectra $\alpha$ and $\beta$, respectively, in inset. The intensity profile (black) of the input pulse is also shown. The intensities are normalized to the peak top values. The exponential tail $I \propto \exp \left(-t / \tau_{0}\right)$ is indicated by a broken line.

due to a band dispersion even in the complete absence of lattice imperfections that cause light scattering. Such a feature can be seen for our PCD, as shown in Fig. 7(d). In contrast to $T_{\text {delay }}$ of the parallel component of PAD, that of the cross component of PAD in Fig. 7(c) increases largely as the frequency approaches the band edge. This increase in $T_{\text {delay }}$ is even larger than that for PCD. Such large delays can be attributed to diffusive transport with multiple scattering, which results in a large increase in the total path length of microwaves.

From the measured complex transmission spectra, we have extracted the response to a pulse input to the PAD. Here, one of the measured spectra of the cross-polarized component for PAD shown in inset in Fig. 8 was multiplied with a Gaussian envelope of width $\Delta f$ centered at $f_{c}$ and then Fourier transformed to give the response to an input Gaussian pulse in the time domain. Note that we used here the complex transmission spectrum, although only its intensity is plotted in the inset. The results with $\Delta f=0.01 \mathrm{c} / d$ and $f_{c}$ $=0.235 \mathrm{c} / d$ ( $\alpha$ in inset), and with $\Delta f=0.01 \mathrm{c} / d$ and $f_{c}$ $=0.315 \mathrm{c} / d(\beta)$ are shown in Fig. 8 by red and green lines, respectively. Here, the input pulse profile is shown by a black line. The transmitted pulses exhibit an exponential tail $I$ $\propto \exp \left(-t / \tau_{0}\right)$ (broken line) $\left(\tau_{0} \approx 1.5 \mathrm{~ns}\right)$ in the delay side, which is typical of diffusive transport; the tail represents the path-length distribution in the diffusive transport.

Consequently, we have successfully explained the peculiar spectral structure in $T_{p}$ for PAD in Fig. 5(a) with the existence of a 3D-PBG and the strong light diffusion outside it. Thus, from this fact, along with the result of $T_{c}$, we finally conclude the experimental substantiation of the formation of an isotropic 3D-PBG in PAD.

\section{B. Photonic eigenstates}

Our PAD has exhibited strongly diffusive characteristics, as described in the preceding subsection, suggesting a possibility of Anderson localization of light. To investigate 

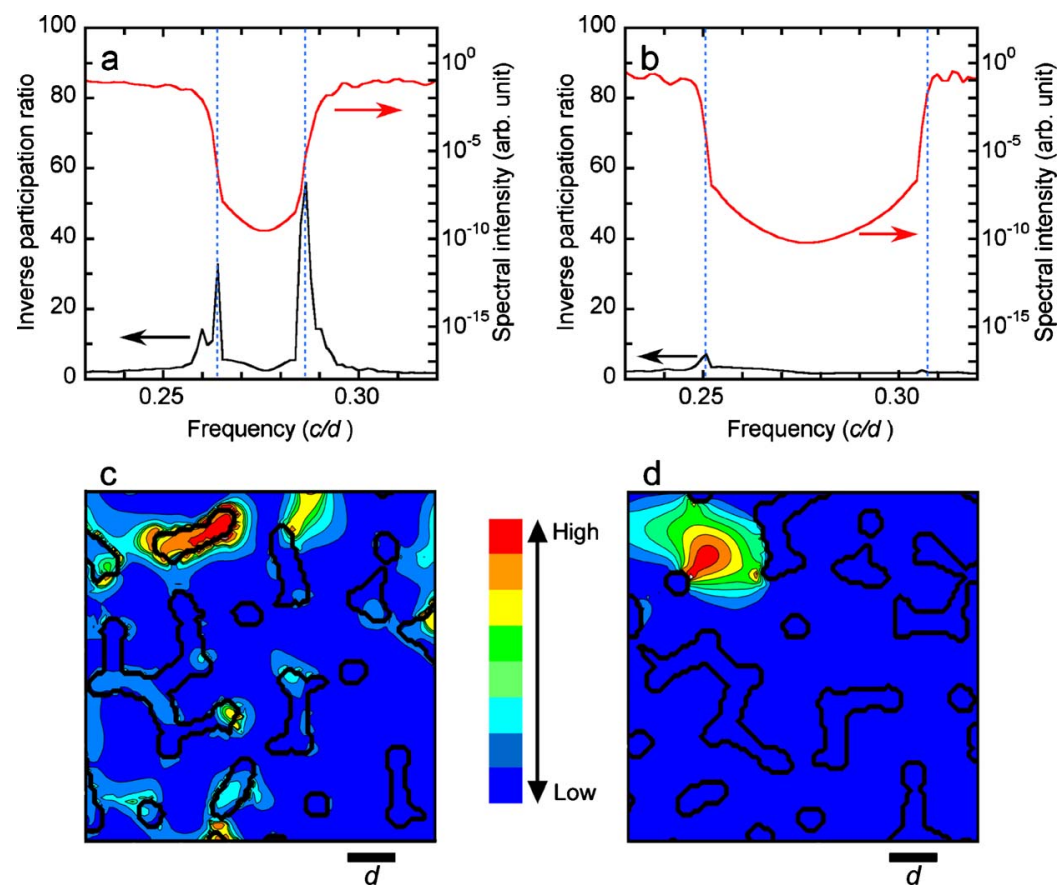

FIG. 9. (Color online) Spectral intensity and inverse participation ratio of (a) PAD and (b) PCD. The mode profiles at $f=0.263 c / d$ and $0.287 c / d$ for PAD, which correspond to the two peaks in inverse participation ratio are shown in (c) and (d), respectively. Here, the regions surrounded by thick black lines correspond to the dielectric components. whether localized states are indeed formed and also to clarify the mechanism of the PBG formation, we numerically examined the photonic eigenstates in our PAD.

In the upper portions of Figs. 9(a) and 9(b), the spectral intensities, which represent PDOS, are presented for PAD and PCD, respectively. Complete 3D-PBGs are clearly observed for both PAD and PCD. These are already presented in Figs. 3(c) and 5(c). To evaluate the degree of localization of the photonic eigenstates, we calculated the inverse participation ratio ${ }^{25}$ (IPR) defined as

$$
\mathrm{IPR} \equiv \frac{\int_{V_{t o t}}|\mathbf{E}(\mathbf{r})|^{4} d \mathbf{r}}{\left(\int_{V_{t o t}}|\mathbf{E}(\mathbf{r})|^{2} d \mathbf{r}\right)^{2}} \cdot V_{t o t},
$$

where $|\mathbf{E}(\mathbf{r})|^{2}$ is the intensity distribution of the electric field in the eigenstate and $V_{t o t}$ is the total volume of the system. When $|\mathbf{E}(\mathbf{r})|^{2}$ is concentrated in a portion $V_{0}$ of the total volume $V_{\text {tot }}\left[|\mathbf{E}(\mathbf{r})|^{2}=\right.$ const $\neq 0$ for $\mathbf{r} \in V_{0}$ and $|\mathbf{E}(\mathbf{r})|^{2}=0$ for $\left.\mathbf{r} \notin V_{0}\right], \mathrm{IPR}=V_{t o t} / V_{0}$. This indicates that IPR $=1$ when the electric-field intensity $|\mathbf{E}(\mathbf{r})|^{2}$ is constant throughout the total volume $V_{\text {tot }}$ and that IPR increases when $|\mathbf{E}(\mathbf{r})|^{2}$ is concentrated in a small volume. Therefore, the IPR can be employed as a measure of the localization of the eigenstates. The calculated IPR values are plotted in the lower portions of Figs. 9(a) and 9(b) for PAD and PCD, respectively. In Fig. 9(a), peaks of IPR are apparent at the band edges for PAD. The mode profiles $|\mathbf{E}(\mathbf{r})|^{2}$ at $f=0.263 c / d$ and $0.287 c / d$, which correspond to the two peaks in IPR for PAD, are shown in Figs. 9(c) and 9(d). These are mode profiles on a two-dimensional cross section cut from the 3D structure. The regions surrounded by thick black lines correspond to the dielectric components. We see that the electric-field intensity $|\mathbf{E}(\mathbf{r})|^{2}$ is concentrated in a small area, thereby exhibiting the feature of localization. It is also apparent that a peak exists at one of the band edges for PCD in Fig. 9(b); however, it is very small and no features of localization was able to be identified in the corresponding mode profiles.

The physical origin of the PBG formation in conventional photonic crystals is often explained in the picture of dielectric and air bands. ${ }^{26,27}$ To examine whether this picture can also be applied to PAD, we have evaluated the concentration factor $(\mathrm{CF})$ of the electric field defined as

$$
\mathrm{CF} \equiv \frac{\int_{V_{\text {air }}}|\mathbf{E}(\mathbf{r})|^{2} d \mathbf{r}}{\int_{V_{\text {tot }}}|\mathbf{E}(\mathbf{r})|^{2} d \mathbf{r}}
$$

for the photonic eigenstates in PAD and PCD. Here, $V_{\text {air }}$ and $V_{\text {tot }}$ represent the air volume and total volume, respectively. If the electric field is distributed evenly for the dielectric and air regions, the concentration factor $\mathrm{CF}$ should equal the volume fraction of the air part, that is, 78\%. The frequency dependences of CF for PAD and PCD are plotted in Fig. 10. At the lower-band top, $\mathrm{CF}$ is approximately $65 \%$ for both PAD and PCD. As the frequency decreases, CF increases and reaches to the volume fraction value, that is, $78 \%$. On the other hand, at the higher-band bottom, CF is approximately $93 \%$, and it decreases gradually with increasing frequency away from the band gap. Typical mode profiles at a frequency near the lower-band top for PAD and PCD are shown in Figs. 11(a) and 11(b), respectively. The tendency of the electric fields concentrating on the dielectric regions is clearly seen in these mode profiles. On the other hand, typical mode profiles at a frequency near the higher-band bottom for PAD and PCD are presented in Figs. 11(c) and 11(d), respectively. Here, in contrast to the mode profiles in Figs. 


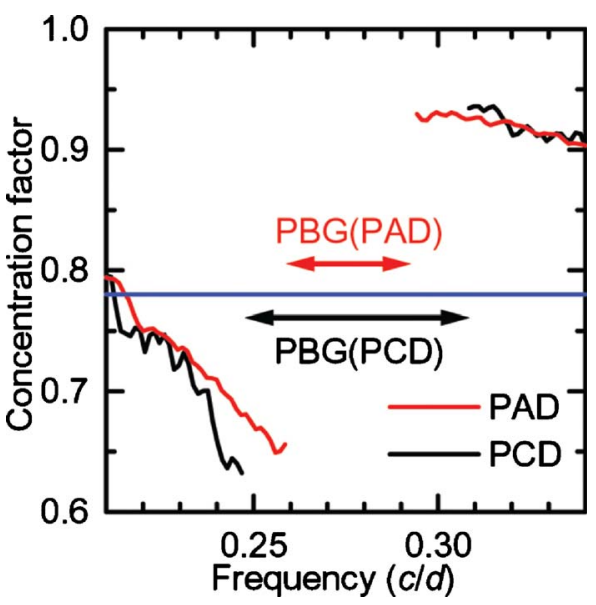

FIG. 10. (Color online) The frequency dependences of the concentration factor of the electric field for PAD and PCD.

11(a) and 11(b), the electric filed instensity is obviously low in the dielectric regions.

\section{DISCUSSION}

As described in Sec. III A, we have experimentally substantiated the formation of an isotropic 3D-PBG in PAD. The isotropic nature of the $3 \mathrm{D}-\mathrm{PBG}$, regardless of the wavevector orientation and polarization direction, should originate in the structural isotropy, and this is a unique feature of PAD. In general, anisotropy in the band gap is inevitable for conventional photonic crystals, as demonstrated for PCD in Figs. 3(a) and 3(b) because their structures are inevitably aniso-
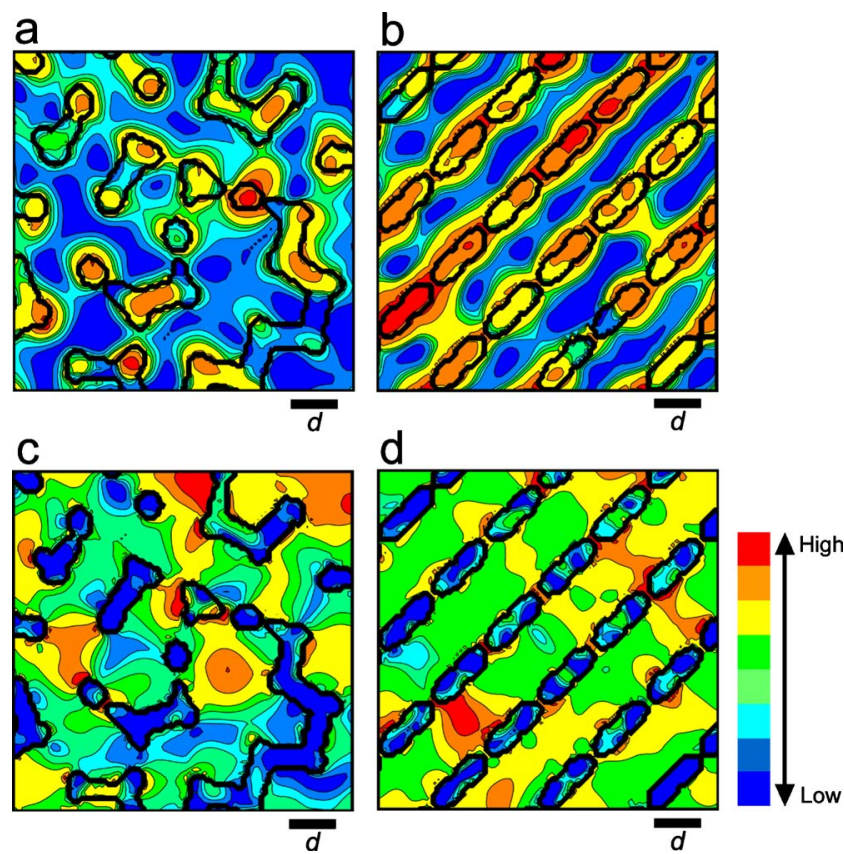

FIG. 11. (Color online) The mode profiles at a frequency near the lower-band top for (a) PAD and (b) PCD, and those at a frequency near the higher-band bottom for (c) PAD and (d) PCD. The frequencies are (a) $f=0.251 \mathrm{c} / d$, (b) $0.240 \mathrm{c} / d$, (c) $0.301 \mathrm{c} / d$, and (d) $0.316 c / d$. tropic. Such anisotropy restricts the orientations of waveguides and other optic devices in designing an optical circuit in photonic crystals. PAD with a completely isotropic 3D-PBG should be free from such restrictions. Furthermore, there is a possibility of realizing a larger $3 \mathrm{D}-\mathrm{PBG}$ than those of conventional photonic crystals by adjusting the structural parameters in PAD because the isotopic structure is, in principle, advantageous in realizing large $3 \mathrm{D}-\mathrm{PBGs}{ }^{7}$

Figures 3(c) and 5(c) show that the gap depth of PAD is nearly the same as that of PCD. Here, it is shown that the density of states at the midgap is lower than that in the passbands by a factor of $10^{-9}$, which is the resolution limit in the present calculations. This fact indicates that the gap in PAD is as clean as that in PCD, with no trace of localized-state formations in the gap. In a previous study, ${ }^{4}$ we investigated by an FDTD calculation the attenuation behavior of an evanescent wave with the midgap frequency when it enters the structure from the surface. Both PAD and PCD showed exponential decay, i.e., $I \propto \exp \left(-r / r_{0}\right) \quad\left(r_{0}\right.$ : the attenuation length) with nearly the same $r_{0}(\approx 0.6 d)$. This fact also indicates that the gap in PAD is as clean as that in PCD. The cleanness of the gap is crucially important when we use the gap in various applications.

In passbands, the PAD has exhibited diffusive light propagation, where the scattering mean-free path decreases significantly as the frequency approaches the band edge, as shown in Fig. 6. The quantity $2 \pi l / \lambda$ reaches three at the band edges, which is only slightly larger than the threshold value of unity in the Ioffe-Regel condition. The quantity $2 \pi l / \lambda$ has been evaluated previously for strongly scattering systems close to the Anderson transition in random media comprising GaAs powders ${ }^{15}(2 \pi l / \lambda=1.5)$, macroporous GaP networks [2.4 (Ref. 22) and 3.2 (Ref. 17)], Ge powders [3 (Ref. 18)], and $\mathrm{TiO}_{2}$ powders [2.5 (Ref. 16) and 5.6 (Ref. 21)]. The value $2 \pi l / \lambda \approx 3$ evaluated for our PAD is comparable to these values.

In Fig. 8, the transmitted pulses exhibit an exponential tail $I \propto \exp \left(-t / \tau_{0}\right)\left(\tau_{0} \approx 1.5 \mathrm{~ns}\right)$ in the delay side, which is typical of diffusive transport. Here, the diffusion constant $D$ can be estimated to be $6.5 \times 10^{4} \mathrm{~m}^{2} / \mathrm{s}$ using the relation: ${ }^{16}$ $1 / \tau_{0}=\pi^{2} D / L^{2}+c /\left(\bar{n} L_{a}\right)$. In general, the diffusion constant can be written as $D=v_{t} l_{t} / 3\left(v_{t}\right.$ : transport velocity; $l_{t}$ : transport mean-free path). Using the values of the scattering mean free path $l$ in Fig. $6\left(l \approx 2.7 d \approx 8 \mathrm{~mm}\right.$ at $f_{c}=0.235 c / d$ and $l$ $\approx 1.7 d \approx 5 \mathrm{~mm}$ at $\left.f_{c}=0.315 \mathrm{c} / d\right)$ for a rough estimation, we obtain $v_{t}=0.08 c-0.13 c$. This is comparable to the transport velocity $v_{t}$ previously evaluated for strongly scattering random media. ${ }^{18,21}$

In PAD, the localized states have been identified at band edges by numerical calculations, as shown in Fig. 9. This result is consistent with the fact that the mean-free path lengths experimentally evaluated at the band edges nearly satisfy the Ioffe-Regel condition. The formation of localized photonic states near the band edges in our PAD is analogous to the formation of localized electronic states in amorphous semiconductors. In principle, sign indicating the localizedstate formation can be detected experimentally, for example, as a deviation from the exponential form of the transmitted pulse tail. ${ }^{16}$ Although we performed analyses similar to those in Fig. 8 for the Gaussian pulses with various frequencies, 


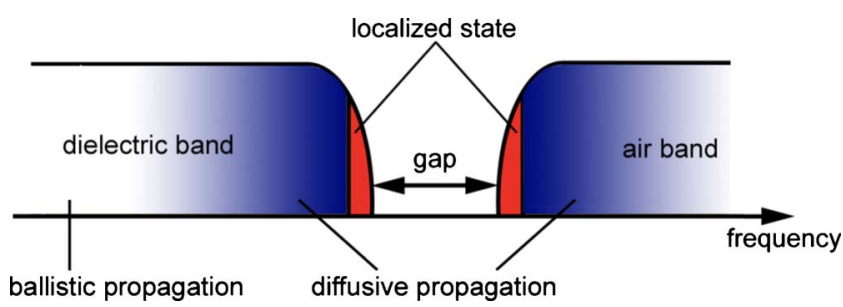

FIG. 12. (Color online) Schematic illustration of the photonic bands and light propagation behavior in PAD.

we failed to detect any clear deviation from the exponential form. This is probably because localized-state formations are limited to considerably narrow frequency ranges.

In textbooks, ${ }^{26,27}$ the physical origin of photonic band-gap formation in photonic crystals is often explained in the picture of dielectric and air bands. Here, let us consider a simple one-dimensional photonic crystal consisting of alternate stacking of dielectric and air layers. At the edge of the Brillouin zone with $k=\pi / a$ ( $a$ : period), the eigenmodes are stationary waves with a wavelength $2 a$. We have two ways to position such a wave so as to conform to the symmetry: in one way the maximum points of $|\mathbf{E}(\mathbf{r})|^{2}$ sit at the dielectric part and in the other they sit at the air part. These two modes should have different eigenfrequencies, leading to the formation of a gap. Figure 10 clearly shows that the picture of the dielectric and air bands can be applied also to the gap formation in PAD, despite the complete absence of lattice periodicity.

In 3D photonic crystals, the structures in which both dielectric and air regions form connected and percolating networks are known to favor the gap formation. ${ }^{67,28}$ Actually, our PAD, as well as PCD, has such a structure. The importance of the connected network structure for gap formation should be related to the vector nature of electromagnetic fields; in order for the field to be confined in dielectric or air region to realize dielectric or air band, each region must be a connected network to accommodate the continuous field lines. ${ }^{26}$ Actually, we have confirmed an extreme fragility of band gap against the segmentation of the dielectric network for PAD and PCD by numerical calculations. When the dielectric network has been segmented, there is no longer continuous pathway to accommodate the whole $\mathbf{E}$ field lines in the dielectric regions; they are forced to penetrate the air regions. This should raise the eigenfrequency at the lowerband top, leading to the closing of the gap.

Figure 10 shows that the frequency dependences of $\mathrm{CF}$ for PAD and PCD are very similar, suggesting that the formation of the dielectric and air bands and thus the formation of the gap in the two structures originate in essentially the same origin. Here, periodicity of the structure in PCD must be irrelevant to the origin. The structural feature common to PAD and PCD is nothing more than a local tetrahedral configuration of dielectric rods, which should be the origin of the gap formation.

The characteristics of photonic bands and light propagation behavior in PAD are summarized schematically in Fig. 12. The PAD has a sizable clean band gap with no trace of localized states inside. The gap is sandwiched between di- electric and air bands. In the dielectric band, PAD exhibits a ballistic light propagation in a low-frequency limit. As the frequency increases, scattering strength increases, and diffusive light propagation becomes dominant in the frequency region close to the band edge. Also above the gap, we have a diffusive light propagation with strong scattering. Localized states are formed at the band edges, where the scattering strength is high enough to nearly satisfy the Ioffe-Regel condition.

\section{CONCLUDING REMARKS}

We fabricated a PAD in a microwave regime, and experimentally confirmed the existence of an isotropic 3D-PBG. We also confirmed that this $3 \mathrm{D}-\mathrm{PBG}$ is clean and does not contain unwanted localized states within it. This proves unambiguously that periodicity is not essential to the realization of a 3D-PBG. The picture of dielectric and air bands in conventional photonic crystals can also be applied to the 3D-PBG formation in PAD as well. The isotropic nature of the 3D-PBG may solve the problem of anisotropy in the conventional 3D-PBG photonic crystals; it can provide a large geometrical flexibility in the applications of photonic devices. The isotropic nature also suggests a possibility of realizing a wider 3D-PBG in future.

Besides the existence of the 3D-PBG, the PAD also showed unique features related to light diffusion and localization. In passbands, the PAD has exhibited diffusive light propagation, where the scattering strength increases significantly as the frequency approaches the band edge. This fact suggests that we may be able to realize a very small light diffuser that can completely eliminate the memory of light polarization and wave-vector orientation with a thickness comparable to the light wavelength. In frequency ranges near the band edge, the scattering strength is high enough to nearly satisfy the Ioffe-Regel condition, which indicates a precursor of light localization. Numerical studies showed that localized states are indeed formed near the band edges. The formation of localized photonic states near the band edges in the PAD is analogous to the formation of localized electronic states in amorphous semiconductors.

These findings indicate that the PAD differs substantially from conventional photonic crystals and random materials studied so far. The PAD provides different insights into the PBG formation mechanism, light diffusion, and localization, and it should open different possibilities in the application of 3D-PBG materials and optical random materials.

\section{ACKNOWLEDGMENTS}

We thank G. T. Barkema of Utrecht Univ. for providing the coordinates of his CRN structure. This work was supported by a Grant-in-Aid for Scientific Research (B) (Project No. 21360027) and a Grant-in-Aid for Scientific Research on Priority Areas "Nano Materials Science for Atomic Scale Modification 474" from the Ministry of Education, Culture, Sports, Science and Technology (MEXT). This work was also supported by the Mitsubishi Foundation and the Sumitomo Foundation. 
*FAX: +81-3-5452-6106; edagawa@iis.u-tokyo.ac.jp

${ }^{1}$ E. Yablonovitch, Phys. Rev. Lett. 58, 2059 (1987).

${ }^{2}$ S. John, Phys. Rev. Lett. 58, 2486 (1987).

${ }^{3}$ M. Notomi, Rep. Prog. Phys. 73, 096501 (2010).

${ }^{4}$ K. Edagawa, S. Kanoko, and M. Notomi, Phys. Rev. Lett. 100, 013901 (2008).

${ }^{5}$ F. Wooten, K. Winer, and D. Weaire, Phys. Rev. Lett. 54, 1392 (1985).

${ }^{6}$ M. Maldovan and E. L. Thomas, Nature Mater. 3, 593 (2004).

${ }^{7}$ C. T. Chan, S. Datta, K. M. Ho, and C. M. Soukoulis, Phys. Rev. B 50, 1988 (1994).

${ }^{8}$ G. T. Barkema and N. Mousseau, Phys. Rev. B 62, 4985 (2000).

${ }^{9} \mathrm{P}$. Sheng, Introduction to Wave Scattering, Localization, and Mesoscopic Phenomena (Academic, San Diego, 1995).

${ }^{10} \mathrm{~A}$. Ishimaru, Wave Propagation and Scattering in Random Media (IEEE Press, New York, 1997).

${ }^{11}$ P. W. Anderson, Philos. Mag. B 52, 505 (1985).

${ }^{12}$ K. Arya, Z. B. Su, and J. L. Birman, Phys. Rev. Lett. 57, 2725 (1986).

${ }^{13}$ C. M. Soukoulis, E. N. Economou, G. S. Grest, and M. H. Cohen, Phys. Rev. Lett. 62, 575 (1989).

${ }^{14}$ N. Garcia and A. Z. Genack, Phys. Rev. Lett. 66, 1850 (1991).

${ }^{15}$ D. S. Wiersma, P. Bartolini, A. Lagendijk, and R. Righini, Nature (London) 390, 671 (1997).

${ }^{16}$ M. Störzer, P. Gross, C. M. Aegerter, and G. Maret, Phys. Rev.
Lett. 96, 063904 (2006)

${ }^{17}$ F. J. P. Schuurmans, M. Megens, D. Vanmaekelbergh, and A. Lagendijk, Phys. Rev. Lett. 83, 2183 (1999).

${ }^{18}$ J. Gómez Rivas, R. Sprik, A. Lagendijk, L. D. Noordam, and C. W. Rella, Phys. Rev. E 63, 046613 (2001).

${ }^{19}$ F. Scheffold, R. Lenke, R. Tweer, and G. Maret, Nature (London) 398, 206 (1999).

${ }^{20}$ A. F. Ioffe and A. R. Regel, in Prog. Semicond., edited by A. F. Gibson, R. E. Burgess, and F. A. Kroeger (Heywood, London, 1960), Vol. 4, p. 237.

${ }^{21}$ M. P. van Albada, B. A. van Tiggelen, A. Lagendijk, and A. Tip, Phys. Rev. Lett. 66, 3132 (1991).

${ }^{22}$ P. M. Johnson, A. Imhof, B. P. J. Bret, J. G. Rivas, and A. Lagendijk, Phys. Rev. E 68, 016604 (2003).

${ }^{23}$ S. Kumar, J. Minerals Metals \& Materials 55, 43 (2003).

${ }^{24}$ C. T. Chan, Q. L. Yu, and K. M. Ho, Phys. Rev. B 51, 16635 (1995).

${ }^{25}$ J. Dong and D. A. Drabold, Phys. Rev. Lett. 80, 1928 (1998).

${ }^{26}$ J. D. Joannopoulos, S. G. Johnson, J. N. Winn, and R. D. Meade, Photonic Crystals-Molding the Flow of Light (Princeton University Press, Princeton, NJ, 2008).

${ }^{27}$ K. Inoue and K. Ohtaka, Photonic Crystals-Physics, Fabrication and Applications (Springer, Germany, 2004).

${ }^{28}$ E. N. Economou and M. M. Sigalas, Phys. Rev. B 48, 13434 (1993). 